\title{
Mars as a compelling target in the continuing search for signs of ancient extraterrestrial life
}

Primary Author:

Andrew Czaja, University of Cincinnati, 513-556-3574, andrew.czaja@uc.edu

Co-Authors (in alphabetical order):

Andrea Corpolongo, University of Cincinnati

Andrew Gangidine, U.S. Naval Research Laboratory

Briony Horgan, Purdue University

Linda Kah, University of Tennessee, Knoxville

Jeff Osterhout, University of California, Los Angeles

Steve Ruff, Arizona State University

Svetlana Shkolyar, NASA Goddard Space Flight Center

Jon Zaloumis, Arizona State University

\section{Co-Signatories:}

Full list available on the MEPAG website at:

https://mepag.jpl.nasa.gov/reports.cfm?expand=decadal 


\section{SUMMARY}

The purpose of this white paper is to document the need to continue our search for ancient life on Mars. Upcoming missions are taking the first steps by directly seeking signs of ancient life with in situ exploration (Mars 2020 and ExoMars) and Mars Sample Return (MSR). However, these missions will not explore the full suite of habitable paleoenvironments on Mars and only a limited geographic region. Whereas a positive result would be a watershed moment for humanity and would compel us to explore the full extent of Mars' biosphere, a negative result would not indicate life never existed on Mars, and additional investigations will be needed. So in either case, as we go forward into the next decade, we will need to target a broader range and distribution of habitable paleoenvironments on Mars, constrain the history of water on Mars, and define a more complete catalog of possible biosignatures, as well as their ubiquity and stability under Mars' preservational conditions. This will also require instrumentation developments that could improve the detection of ancient biosignatures during future robotic and human missions.

\section{INTRODUCTION}

Mars has been and continues to be a compelling target for astrobiology studies. Although the goal of finding life on Mars has been around at least since Schiapparelli and Lowell made telescopic observations of its surface in the late 19th century (and probably long before that), the 1996 announcement of evidence of supposed past life in martian meteorite ALH84001 (though later refuted), greatly accelerated the search. This began with the search for aqueous alteration and other evidence of formerly habitable environments with orbiters, landers, and rovers (e.g., MRO, OMEGA, Spirit, Opportunity, Curiosity, Phoenix).

Because of the physical similarities between Earth and Mars, the evidence for stable liquid water on the surface of each planet early in their respective histories, and the fact that evidence for life on Earth dates back approximately 3.5 billion years to the age of the earliest well-preserved sedimentary rocks, and to a time when environments of Earth and Mars were likely very similar, there is a chance that life was also an element of the Noachian environment of Mars. Finding evidence of life beyond Earth is one of the major goals of NASA's planetary exploration programs. Mars in particular is a key target for the search. Finding such evidence would have major implications for science as well as society.

Mars' current surface conditions are extremely challenging for life as we know it to survive (carbon-based life requires liquid water as a medium for biochemical reactions), let alone thrive. However, if we envision the diversity of environments where life occurs on Earth - from deep in the crust to the cores of nuclear reactors - it is reasonable to assume that if life ever established itself on or near the surface of Mars, it may still persist in deep habitable refuges, assuming the surface environment changed to its current conditions gradually enough that life could adapt and spread to subsurface locations that are more clement to life. The search for martian biosignatures focuses largely on the search for evidence of past life rather than extant life for multiple reasons. First, accessing the subsurface of Mars is more difficult than the surface. Drilling more than a couple of meters without heavy equipment and drilling fluid is not currently feasible with robotic spacecraft. Another hurdle to overcome relates to planetary protection, i.e., the challenge of not contaminating the subsurface of Mars and not containing any indigenous life we might find should we want to return a sample to Earth. Finally, we have yet to develop a clear search protocol for subsurface targets. 


\section{THE SEARCH FOR EVIDENCE OF ANCIENT LIFE ON MARS SO FAR}

Possible ancient biosignatures on/near the martian surface. The number of possible ancient biosignatures that could exist on/near the surface of Mars is large and has been described in detail in several recent publications (e.g., Hays et al., 2017; Onstott et al., 2019; Beaty et al., 2019; MEPAG Goals Document, 2020) so here we will provide only a brief overview. Plausible ancient martian biosignatures are argued to be similar to the types of microbial biosignatures we can find in the ancient rock record of Earth (McMahon et al., 2018). These include morphologically preserved organic-walled microbial body fossils, fossilized macroscopic microbial structures (i.e., stromatolites, microbialites, and microbially induced sedimentary structures), trace fossils such as microbial borings, and geochemical biosignatures.

Microbial body fossils have been identified throughout Earth's fossil record (e.g., Schopf, 2006) and provide strong evidence for the early origin of life here, and are crucial for ascertaining a biological origin from potential fossils recovered from the geologic record. However, such remains are often contentious due to variable preservation combined with the simplicity of morphological remains, which can be easily distorted by post-depositional processes. Macroscopic structures such as stromatolites of presumed biological origin are common in the ancient record, though these are sometimes more controversial due to the lack of co-occurring microbial body fossils and the ability of certain stromatolite morphotypes to form abiologically, particularly in the early rock record (Grotzinger \& Rothman, 1996, Grotzinger \& Knoll, 1999, McLoughlin et al., 2008). Pseudofossils can similarly be mistaken for microbial remains because they mimic the size and morphology of microfossils, though likely arise through completely abiotic origin (e.g., Javaux, 2019, and references therein).

Geochemical biosignatures are also commonly used to characterize the identity, distribution, and evolution of life on Earth. These types of biosignatures include high concentrations of organic matter, labile molecular fossils (e.g., hopanoids) that may indicate the presence of specific groups of organisms, isotopic compositions of sedimentary organic matter (kerogen, bitumens) and redox sensitive elements in minerals, as well as elemental ratios and trace element concentrations (e.g., Summons et al., 1994; Simoneit et al., 2004; Brocks et al. 2005; Schopf et al., 2005; Havig et al., 2011; Gangidine et al., 2020). Such biosignatures are most convincing when multiple types agree with a biological interpretation in a "cascade of evidence". Ideally, these chemical biosignatures can be applied in conjunction with physical remains to convincingly argue for a biological origin. Such an approach using multiple lines of evidence would no doubt be required to determine the presence of life on returned Mars samples.

Paleoenvironments that could preserve ancient biosignatures. In terms of where to search for evidence of ancient life on Mars, we have only begun to scratch the surface. With the exception of the Viking missions, there has not been a mission to search for evidence of past or present life on Mars. The MER missions and the current MSL mission have explored formerly habitable environments, namely two crater lakes (Gusev and Gale craters by Spirit and Curiosity, respectively), a hydrothermal system (the Home Plate region within Gusev crater by Spirit), and a region altered by liquid water in the past (Meridiani Planum, by Opportunity). The upcoming Mars 2020 (Perseverance) mission will explore Jezero crater, another former crater lake, and the ESA ExoMars mission will explore Oxia Planum, a formerly water-altered region within a catchment containing significant amounts of clays. These are all excellent locations to search for evidence of past life, but are not the only habitable paleoenvironments. Thus, there is a need to 
build off of past successes and broaden our search to as many paleoenvironments as possible, including ancient fluvial channels and valley networks, hydrothermal settings associated with either volcanic regions or impact heating, paleosols, subaqueous hydrothermal systems, and subsurface hydrothermal systems (e.g, Hays et al., 2017; Onstott et al., 2019).

Recommendation. NASA's Mars 2020 mission and planned follow-up MSR mission will explicitly search for signs of ancient life on Mars. A positive result would provide a watershed moment for humanity and mean our next goal should be to explore the full extent of the martian biosphere. However, a negative result from these missions would not indicate the absence of life on Mars because they will not explore the full range of habitable paleoenvironments or regions on Mars. Either way, we need to plan for the exploration of additional paleoenvironments, and continue to hone our search strategies and techniques, as described in sections 3 and 4, below.

\section{ASPECTS OF BIOSIGNATURES REQUIRING FURTHER ATTENTION}

Although our understanding of which biosignatures to expect on Mars and how to find them has progressed in recent years, as thoroughly documented by Hays et al. (2017) and Onstott et al. (2019), there are still many unknowns. Below is a non-exhaustive list of knowledge gaps that, if resolved, could greatly aid our search for evidence of ancient life on Mars.

History of water on Mars. There is ample and convincing evidence for the presence of persistent bodies of water (e.g., lakes and rivers) on and below the surface of Mars in the Noachian and Hesperian. However, it is not yet known whether Mars was habitable globally, or if habitability was confined to relatively narrow niche environments (e.g., hot springs, crater lakes, subsurface environments). Understanding the former distribution, timing, and duration of liquid water on Mars will greatly increase our understanding of the formation of habitable environments and will help focus the search for ancient Martian biosignatures.

Understand the stability and ubiquity of physical and chemical biosignatures. For the biosignatures listed in section 2, their ability to be preserved in geologically and/or geochemically distinct environments throughout time is not fully understood. Both physical and chemical biosignatures may be affected or destroyed by diagenetic processes such as mineral replacement, dissolution and recrystallization, and transformation of host rock mineral phases (e.g., opal to quartz) among others. Previous work combining field and lab analyses of Mars analog materials has shown that detailed studies of biosignature preservation potential based on paleoenvironments, lithologies, and post-depositional conditions will be extremely useful for predicting where we might find robust biosignatures on Mars (e.g., Westall et al., 2015; Shkolyar and Farmer, 2018), and more such studies are needed. Such studies are also critical to develop protocols for distinguishing true biosignatures from potential biomorphic (i.e., false) signatures. Combined, these predictions can be used to aid landing site selection for future missions, to determine which regions of interest within a landing site contain lithologies that should be prioritized for exploration, and to provide in situ protocols to triage potential targets of interest.

Understand the taphonomy of biosignatures in Mars analog materials under Mars-like conditions. Some of these materials include carbonate, shale, sulfate, serpentinite, and siliceous sediments (e.g., sinter deposits, cherts). Although taphonomic processes (i.e., morphological and chemical changes that take place either before or during the processes of fossilization) are relatively well-characterized for some lithotypes, little is known for others because of factors such as higher solubility, softness, and overall lack of representation in Earth's ancient rock 
record. Mars has had a very different geologic history with very different surface conditions compared to those of Earth. Because Mars is understood to lack plate tectonics and has had much more limited hydrologic activity as compared to Earth, we must examine the preservation potential of lithotypes that may have persisted on Mars' surface for billion-year timescales that would not be expected in Earth's active surface environment. Additionally, Mars has experienced markedly different atmospheric conditions, and has since exhibited significant loss of its atmosphere, as well as a high flux of surface radiation following the loss of its magnetic field. Experimental taphonomic studies investigating the stability of biosignatures in Mars-like atmospheric and surface radiation conditions are critical to place constraints for targeting.

Presence and distribution of sedimentary carbonates. Carbonate minerals play a major role in the preservation of biosignatures on Earth, even in units deposited prior to the origin of skeletonizing organisms (e.g., microbial textures and organic matter in stromatolites). We also understand the chemistry of carbonate deposition, both chemical and biochemical on Earth. It is expected that carbonate deposits should exist on Mars as well, but to date, virtually all significant carbonate deposits detected, whether from orbital or rover analyses, are interpreted to have formed from the alteration of olivine-rich igneous material with the exception of possible lacustrine carbonate in Jezero crater (Horgan et al., 2020). Locating sedimentary carbonate (or documenting its absence) is important for the search for biosignatures.

Understand the geologic context and history of silica deposits on Mars. Hydrated silica deposits may be ubiquitous on Mars (Sun and Milliken, 2018) and have been the subject of great interest in the astrobiology community because of their high preservation potential for microbial remains on Earth (e.g., Farmer and Des Marais, 1999). For example, certain features in the hydrated silica deposits discovered by the Spirit rover in the Columbia Hills region of Gusev crater resemble microbially-mediated structures in hot spring deposits on Earth (Ruff and Farmer, 2016). Constraining the paleoenvironment and the local geochemical conditions in which such deposits form would help to constrain expected geochemical signatures and potential biosignatures. Similarly, it will be necessary to determine the origin of hydrated silica associated with ancient crater lakes such as in Jezero crater (Tarnas et al., 2019). Hot spring sinter deposits on crater rims caused by impact-induced hydrothermal activity have been identified on Earth (Osinski et al., 2013), although no such deposits have yet been identified on Mars, but the vast number of preserved impact craters on Mars makes this a compelling location to search.

\section{INSTRUMENT DEVELOPMENT TO AID IN THE SEARCH}

Although the search for ancient life on Mars has been aided by astounding technological advances and instrument miniaturization for use on orbiters, rovers, and landers, additional innovation will be necessary to continue the search, particularly for future in situ missions, both robotic and human. It is likely that others have thought of some if not all of these ideas already, but the purpose here is to be sure that they are documented, and particularly for the purpose of searching for ancient life. This is not an exhaustive list of instrumentation recommendations that would aid in the search, but serves as a "call-to-arms" to the community.

An increased focus on large-scale, high resolution mapping and mineralogical surveys of Mars' surface. This approach would greatly advance all aspects of Mars exploration by allowing for increased knowledge of the geology of Mars. In terms of the search for ancient life, it would specifically allow for the identification of additional habitable paleoenvironments with 
higher confidence in their interpretations. This could potentially be achieved with two complementary mission types: higher resolution orbital payloads and the drone missions.

HiRISE and CRISM aboard the Mars Reconnaissance Orbiter have provided impressive high-resolution imagery and mineralogical data, respectively, but because the resolution comes with a tradeoff in aerial coverage, only relatively small areas of the surface have thus far been imaged. We are confident that imaging teams are working on ways to enhance resolution further and also to increase aerial coverage, and we would like to encourage this effort. Perhaps an additional effort could include a constellation of imaging and spectroscopic satellites for enhanced coverage.

Another mission type could follow on from the hopefully successful technology demonstration of Ingenuity, the helicopter accompanying Perseverance as part of the Mars 2020 mission. A mission could be developed that would consist of a fleet of mapping drones to image and measure mineralogy of a large number of carefully selected localities on Mars and produce data at the outcrop scale. This would fill a gap in resolution between orbital payloads and rovers that could greatly increase our confidence in the geology, habitability, and astrobiological potential of proposed landing sites and increase the likelihood of mission success, whether a rover or human mission.

Small scale "grab-and-go"-style MSR missions. Building on the previous suggested developmental need, if the confidence in a given site becomes high enough based on increased knowledge from orbital- or drone-based payloads, for example if these types of missions revealed outcrop scale morphological biosignatures like microbialites with the mineralogy expected for such biologically-mediated structures, one could envision a Mars Sample Return mission that would target such a site directly without an additional landed caching mission. Based on past successful rover missions to Mars, we have obtained detailed outcrop scale knowledge of a few locations that certainly were habitable in the Noachian/Hesperian, and might even possess materials that could host biosignatures. For example, the potential biosignatures observed among the hydrated silica deposits in the Columbia Hills (Ruff and Farmer, 2016) were sufficiently compelling to help make this location a finalist landing site for the Mars 2020 mission. Another site of high astrobiological and preservation potential is Gale crater, where, for example, silicified mudstones were discovered by the Curiosity rover (Hurowitz et al., 2017).

Increased capabilities of ground-based instruments for detecting biosignatures. Such developments could include increased magnification of microscopic imaging systems for the direct detection, in situ, of micron-scale microbial fossils of all types (e.g., compressions, impressions, cast/mold). While this capability would be useful in its own right (and also for measuring grain sizes of fine-grained sediments), it would be even more so when paired with the ability to produce petrographic sections of rock by cutting and polishing. These sections could be analyzed with transmitted light microscopy to search for 3-D preserved microscopic fossils embedded in a transparent mineral matrix. A large number of technological hurdles would need to be overcome to make thin sectioning on Mars possible. It is a power-intensive process and also generally requires the use of a liquid coolant (typically water). Thus, this ability may need to wait for human missions to Mars, where large amounts of water and power would be needed.

The detection of geochemical biosignatures is perhaps more likely than microbial body fossils, which, being soft-bodied, tend to degrade easily and thus have a lower preservation potential. Therefore, the further development of field deployable instruments that can measure 
elemental and/or isotopic ratios with high precision and accuracy, as well as with high spatial and spectral resolution would be revolutionary. The Mars 2020 Perseverance rover will include two Raman spectrometers (Vis-Raman on SuperCam and UV Raman on SHERLOC) for the determination of mineralogy and detection of trace organic components, but these instruments are limited both in terms of spectral coverage and spatial resolution. Perseverance will also include an X-ray fluorescence spectrometer and mapper (PIXL) that will allow for quantitative measurements of elemental abundance, which will be used to study the composition of rocks and can detect elemental abundances that could suggest the influence of life. Such instruments are revolutionary and the next generation of these will hopefully include even greater elemental coverage, lower detection limits and a finer spatial resolution so that we can have greater confidence in our biosignature interpretations. The continued development of miniaturized mass spectrometers similar to those for ESA's ExoMars and lunar PROSPECT missions (Goesmann et al., 2017; Sefton-Nash et al., 2020) for measuring stable isotopic ratios (e.g., C, H, S, N, O, Fe, Mo) of solid material would also be revolutionary for the detection of biosignatures, particularly if they could measure isotopes of redox-sensitive transition metals.

Increased capabilities for accessing the subsurface. Another technological advancement relates to sample acquisition. Because of the highly oxidizing and high radiation environment of Mars' surface, drilling beneath the affected zone $(\sim 2 \mathrm{~m})$ is desired, but drilling more than a few centimeters into consolidated rock without lubricant is a great challenge. Also, the typically required rotary percussive drilling is highly damaging to weaker lithologies. Therefore, developments in drilling technology that allows for drilling to a depth of $>2$ meters without lubricant, and can also provide intact drill core from these depths would be highly beneficial. This is surely a tremendous challenge and would be more easily included on a human mission due to the presumably less restrictive mass and energy allowances of such a mission. Another intriguing possibility for studying the subsurface that has been proposed would be to explore martian caves, which could naturally protect organics, with airborne drones (Wiens et al., 2020).

Limiting contamination during sampling. With all of these new technologies, ways of further limiting/eliminating elemental, biological, and organic contaminants would be necessary because of the increased resolution and detection limits called for. Methods for acquiring very high-resolution contamination knowledge would also be needed when contamination control is not fully possible.

Improvements in Earth-based analytical capabilities. Developments in Earth-based laboratory instruments would also help, but current instruments are already well ahead of anything sent on a mission. One possible example is in isotope mass spectrometry for both isotopes and elemental ratios. A separate white paper was submitted about this topic to the Planetary Science and Astrobiology Decadal Survey. Plans and perhaps technological advancements are needed for sample handling and storage of returned samples, for both planetary protection and sample contamination concerns. Such planning is underway for the proposed return of samples collected by the Perseverance rover, and this planning should be encouraged and supported.

\section{CONCLUSIONS}

Our understanding of Mars as a planet and its habitability have greatly increased in the last two decades with a concerted effort on the part of NASA and other space agencies. Despite 
this increased understanding, there are still many open questions and of course the largest still remains: "Did Mars ever host life?" We have also only begun to scratch the surface in terms of numbers of locations and types of habitable paleoenvironments searched. Thus, the search needs

to be continued and we support direct analyses of Mars to understand its history and locations of habitable environments, further development of our understanding of what biosignatures to look for and how they are preserved, as well as technology development for increased ability to detect biosignatures during robotic and proposed human missions. Such developments will also aid in our search for and understanding of the evolution of life on Earth. The lessons learned from the study of Mars and biosignatures in general-as well as the technologies developed for these tasks-will be of broader benefit to science and humanity, and can also be utilized in the search for life elsewhere in the solar system (and someday, beyond).

\section{REFERENCES}

Beaty D. et al., 2019, Meteorit. Planet. Sci. 54(S1): S3-S152

Brocks, J.J., et al., 2005, Treatise on Geochemistry, 8: 63-115.

Djokic, T. et al., 2017, Nature Comms., 8(1): 1-9.

Farmer, J.D. and Des Marais, D.J., 1999, JGR: Planets, 104(E11): 26977-26995.

Gangidine, A., et al., 2020, Astrobiology, 20(4): 525-536.

Goesmann, F., et al., 2017, Astrobiology, 17(6-7): 655-685.

Grotzinger, J. P., and Rothman, D., 1996, Nature, 383(6599): 423-425.

Grotzinger, J. P., and Knoll, A. H., 1999. Ann. Rev. Earth Planet. Sci. 27(1): 313-358.

Havig, J.R. et al., 2011, JGR: Biogeosciences, 116 (G1): G01005

Hays, L.E. et al., 2017, Astrobiology, 17(4): 363-400.

Horgan, B.H.N. et al., 2020, Icarus, 339: 113526

Hurowitz, J.A. et al., 2017, Science, 356: eaah6849.

Javaux, E.J., 2019, Nature, 572(7770): 451-460.

McLoughlin, N. et al., 2008, Geobiology, 6(2): 95-105.

McMahon, S. et al., 2018, JGR: Planets 123(5): 1012-1040.

MEPAG, Mars Scientific Goals, Objectives, Investigations, and Priorities: 2020. D. Banfield, ed., https://mepag.jpl.nasa.gov/reports.cfm.

Onstott, T.C. et al., 2019, Astrobiology, 19(10): 1230-1262.

Osinski, G.R. et al., 2013, Icarus, 224(2): 347-363.

Ruff, S.W. and Farmer, J.D., 2016, Nature Comms., 7(1): 1-10.

Schopf, J.W. et al., 2005. Astrobiology, 5(3): 333-371.

Schopf, J.W., 2006, Phil. Trans. R. Soc. B, 361: 869-885.

Sefton-Nash, E., et al., 2020, 51st Lunar and Planetary Science Conference, Abst. \#2367

Shkolyar, S. and Farmer, J., 2018, Astrobiology, 18(11): 1460-1478.

Simoneit, B.R., 2004, Adv. Space Res., 33(8): 1255-1261.

Summons, R.E. et al., 1994, Geochim. Cosmochim. Acta, 58(13): 2853-2863.

Sun, V.Z. and Milliken, R.E., 2018, Geophy. Res. Lett., 45(19): 10,221-10,228.

Squyres, S.W., et al., 2006, JGR: Planets 111(E2): E02S11

Tarnas, J.D. et al., 2019, Geophys. Res. Lett., 46(22): 12771-12782.

Westall, F. et al., 2015, Astrobiology, 15(11), 998-1029.

Wiens, R.C. et al., 2020, 3rd International Planetary Caves Meeting (LPI Contrib. No. 2197) 